\title{
Cyber-Medical Systems: Challenges and Possibilities
}

\author{
Levente Kovács \\ Physiological Controls Research Center, University Research and Innovation Center \\ Óbuda University, Budapest, Hungary \\ kovacs.levente@nik.uni-obuda.hu \\ PLENARY PAPER
}

\begin{abstract}
The goal of the plenary talk is to give an overview on the one of the most dynamically changing research topic: cyber-medical systems.
\end{abstract}

By the needs of Industry 4.0 digitalization of industry has been accentuated. However, cybersphysical systems left its footprints in healthcare as well, formalizing the cyber-medical system requirements: how to help everyday life of medical doctors and patients by using medical devices in a more personalized way.

This concept meshes the entire biomedical engineering research field. The idea is to create mathematical algorithms able to be personalized on the patients' need and physiology, use cloud computation techniques to fasten the decision support and big data analysis for feature extraction. As a result, the cyber-medical system concept is equivalent with a smart healthcare framework where by using the computational power possibilities together with machine learning, artificial intelligence and control engineering methods we would like to intensify the decision support of doctors, nurses and patients in order to use in an intelligent way the knowledge-based medical applications.

The presentation will give an overview on the above mentioned aspects: how the cyber-medical system concept appeared, what the trends are, how it is influencing our everyday's life including education, research, medical industry and healthcare sector. In order to give concrete examples, individualized model-based applications are presented such as:

- Artificial pancreas problem: Recent technological advances in diabetes treatment like Continuous Glucose Monitors (CGMs) for the subcutaneous measurement of glucose concentration and insulin pumps for the subcutaneous delivery of insulin allowed investigating the applicability of an external controller. In type 1 diabetes, where the disease can be characterized as a general clinical picture (e.g. complete pancreatic $\beta$-cell insufficiency) different individualized model-based (mostly model predictive control (MPC) based) solutions have been already formulated and even clinical trials appeared demonstrating its applicability. However, closing the loop needs the integration of individualized control methods and robust control algorithms in order to successfully reject hypoglycemic episodes. Moreover, the scientific community started to transpose the problem on type 2 diabetes as well, trying to cover the largest diabetic population with the artificial pancreas concept.

- $\quad$ Tamed cancer concept: Beside general cancer therapies like chemotherapy, targeted molecular therapies appeared in order to focus on a given mechanism of the tumor growth stopping it in a more effective / individualized way. Antiangiogenic therapy is one targeted molecular therapy arose in the last decade which aims to stop tumor angiogenesis, i.e. formation of new blood vessels; hence, minimizing the tumor's volume. However, the corresponding drugs are very expensive, and in high doses may have side effects; moreover, the currently used clinical protocols are determined empirically. As a consequence, this problem can be seen as a double optimal control problem: on the one hand the aim is to minimize the tumor's volume by a model-based control algorithm, but it is also crucial to inject the corresponding inhibitor (drug) in an optimal way (minimizing costs). Due to the heterogeneous nature of the patients by a recently gained ERC StG grant of the European Union we try to develop a novel concept: taming cancer. Beside creating a more realistic tumor growth mathematical model, we would like to decrease the injection sampling time of inhibitors that combined with a robust control method can control the tumor volume artificially and in this taming it. As a result it would be even possible living with the tumor in a controlled way. 
L. Kovács • CyberMedical Systems: Challenges and Possibilities 